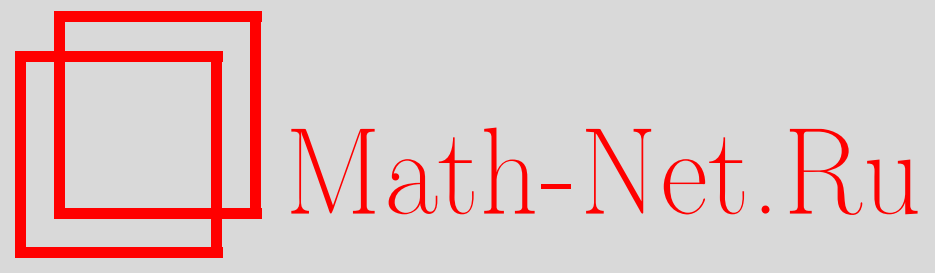

В. Г. Овчинников, K динамическому программированию по значениям в полугруппе, Вестн. Сам. гос. техн. ун-та. Сер. Физ.-мат. науки, 2016, номер 1, 158-166

DOI: https://doi.org/10.14498/vsgtu1473

Использование Общероссийского математического портала MathNet.Ru подразумевает, что вы прочитали и согласны с пользовательским соглашением

http: //www . mathnet.ru/rus/agreement

Параметры загрузки:

IP : 54.197 .130 .99

26 апреля 2023 г., 16:14:29

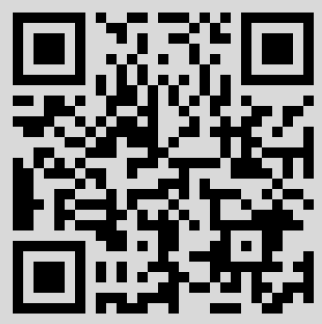


УДК 519.7

\title{
К ДИНАМИЧЕСКОМУ ПРОГРАММИРОВАНИЮ ПО ЗНАЧЕНИЯМ В ПОЛУГРУППЕ
}

\section{В. Г. Овчинников}

Самарский государственный технический университет, Россия, 443100, Самара, ул. Молодогвардейская, 244.

\begin{abstract}
Аннотация
Для не рассматривавшейся ранее со значениями целевой функции в линейно упорядоченной абелевой полугруппе $P$ задачи дискретного оптимального управления даются характеризация разрешимости и на ее основе алгоритм, ищущий оптимальный процесс, используя доставляющие значения Беллмана элементы ограничивающих множеств. Отмечаются модификации данного алгоритма, когда

1) $P$ - непустое естественно упорядоченное подмножество чисел с операцией получения максимума из двух чисел;

2) $P$ - естественно упорядоченное множество неотрицательных чисел со сложением (умножением);

3) $P$ - лексикографическое произведение $m$ (не менее двух) линейно упорядоченных абелевых полугрупп;

4) $P$ - лексикографическое произведение $m$ (не менее двух) множеств вещественных чисел с естественным порядком и сложением, и данный алгоритм получает $m$ - оптимальный процесс проще, чем предыдущий алгоритм автора.
\end{abstract}

Ключевые слова: линейно упорядоченная абелева полугруппа, дискретное оптимальное управление, оптимальный процесс, доставляющие значения Беллмана элементы ограничивающих множеств, динамическое программирование, лексикографические произведения, алгоритмы.

1. Статья продолжает исследования применения динамического программирования $[1,2]$. В ней для упрощения алгоритма [2] используется не рассматривавшаяся ранее в общей формулировке и в указанных ниже частных случаях следующая задача дискретного оптимального управления.

ЗАДАчА $\mathfrak{A}$. Пусть известно следующее:

- пространства $A, B$;

- множество шагов $T(1<|T|<\infty)$, наделенное строгим порядком $\prec$,

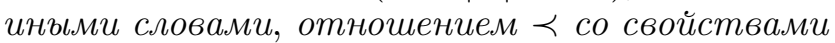

$$
(i \prec j) \Rightarrow(i \neq j)(\forall i, j \in T), \quad(i \prec g) \wedge(g \prec j) \Rightarrow(i \prec j)(\forall i, g, j \in T),
$$

(C) 2016 Самарский государственный технический университет.

\section{Образец для цитирования}

О в чи нн иков В. Г. K динамическому программированию по значениям в полугруппе // Вестн. Сам. гос. техн. ун-та. Сер. Физ.-мат. науки, 2016. Т. 20, № 1. С. 158-166. doi: 10.14498 /vsgtu1473.

\section{Сведения об авторе}

Валерий Гаврилович Овчинников (ovg.samara@mail.ru), старший преподаватель, каф. разработки нефтяных и газовых месторождений. 
имеющее подмножество начальных шагов

$$
T_{0}=\{i \in T \mid \emptyset=\{j \in T \mid j \prec i\}\}(\neq T),
$$

обозначаемые $\{\pi(i)\}$ одноэлементные подмножества

$$
\{j \in T \mid(j \prec i) \wedge(\emptyset=\{g \in T \mid j \prec g \prec i\})\}\left(\forall i \in T \backslash T_{0}\right)
$$

и удовлетворяющее равенству $\emptyset=M \cap T_{0}$ подмножество финальных шагов

$$
M=\{i \in T \mid \tau(i)=\emptyset\},
$$

где $\tau(i)=\left\{j \in T \backslash T_{0} \mid \pi(j)=i\right\}$;

- функиии $X, U, S$ с конечными множественными значениями

$$
\begin{gathered}
X(i) \subset A(\forall i \in T), \quad U(i, \alpha) \subset B\left(\forall i \in T \backslash T_{0}\right)(\forall \alpha \in A), \\
S(i, \alpha, \beta) \subset A(|S(i, \alpha, \beta)| \leqslant 1)\left(\forall i \in T \backslash T_{0}\right)(\forall \alpha \in A)(\forall \beta \in B) ;
\end{gathered}
$$

- обозначение $D$ множества называемых процессами пар $(x, u)$ функиий $x, u$ со значениями $x_{i} \in A(\forall i \in T), u_{i} \in B(\forall i \in T)$ при ограничениях

$$
\begin{array}{cl}
x_{i}=u_{i}\left(\forall i \in T_{0}\right), & x_{i} \in X(i)(\forall i \in T), \quad u_{i} \in U\left(i, x_{\pi(i)}\right) \quad\left(\forall i \in T \backslash T_{0}\right), \\
& x_{i} \in S\left(i, x_{\pi(i)}, u_{i}\right) \quad\left(\forall i \in T \backslash T_{0}\right)
\end{array}
$$

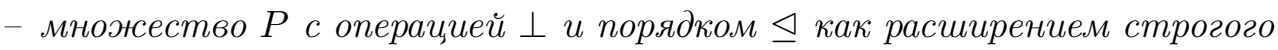
порядка по правилам

$$
\left(p_{1} \unlhd p_{2}\right) \Leftrightarrow\left(p_{1} \triangleleft p_{2}\right) \vee\left(p_{1}=p_{2}\right)\left(\forall p_{1}, p_{2} \in P\right),
$$

являющееся линейно упорядоченной абелевой полугруппой $\langle P, \perp, \unlhd\rangle$, таким образом, имеющее свойства

$\left(p_{1} \perp p_{2}\right) \perp p_{3}=p_{1} \perp\left(p_{2} \perp p_{3}\right)\left(\forall p_{1}, p_{2}, p_{3} \in P\right)($ ассоииативность $\perp)$, $p_{1} \perp p_{2}=p_{2} \perp p_{1}\left(\forall p_{1}, p_{2} \in P\right)($ коммутативность $\perp)$,

$\left(p_{1} \unlhd p_{2}\right) \vee\left(p_{2} \unlhd p_{1}\right)\left(\forall p_{1}, p_{2} \in P\right)($ линейность $\unlhd)$,

$\left(p_{1} \unlhd p_{2}\right) \Rightarrow\left(p_{1} \perp p_{3}\right) \unlhd\left(p_{2} \perp p_{3}\right)\left(\forall p_{1}, p_{2}, p_{3} \in P\right) \quad($ стабильность $\unlhd$ относительно $\perp)$;

- обозначение обобщённой операции $\frac{1}{i \in I}$ с результатом $\frac{1}{i \in I} q(i)$ как элементом $\Lambda(|I|, \omega, q) \in P$, определяемым по функиии

$$
q: I \rightarrow P(\emptyset \neq I \subset T)
$$

из условий

$$
\begin{gathered}
k>1 \Rightarrow \Lambda(k, \omega, q)=\Lambda(k-1, \omega, q) \perp q(\omega(k))\left(\forall k \in N_{|I|}\right), \\
\Lambda(1, \omega, q)=q(\omega(1))
\end{gathered}
$$

независимо от выбора биекиии

$$
\omega: N_{|I|} \rightarrow I \quad\left(N_{m}=\{1, \ldots, m\}(\forall m \in \mathbb{N})\right)
$$

(ср. с замечаниями N.B. [3, с. 62]); 
- затрать шагов

$$
f(i, \gamma, \alpha, \beta)(\in P)\left(\forall i \in T \backslash T_{0}\right)(\forall \gamma, \alpha \in \mathrm{A})(\forall \beta \in B) ;
$$

- обозначение $\min _{\gamma \in \Gamma}(\unlhd) Q(\gamma)$ наименьшего по порядку $\unlhd$ значения фуункии

$$
Q: \Gamma \rightarrow P
$$

Требуется найти называемую оптимальным процессом пару $\left(x^{*}, u^{*}\right) \in D$, удовлетворяющуюо равенству

$$
\rfloor_{i \in T \backslash T_{0}} f\left(i, x_{i}^{*}, x_{\pi(i)}^{*}, u_{i}^{*}\right)=\min _{(x, u) \in D}(\unlhd) \perp_{i \in T \backslash T_{0}} f\left(i, x_{i}, x_{\pi(i)}, u_{i}\right) .
$$

ЗАМЕчАНИЕ 1. Если

$$
P \subset W \in\{\mathbb{R}, \mathbb{Q}, \mathbb{Z}, \mathbb{N}\}(1<|P|), \quad p \perp q=\max (p, q)(\forall p, q \in P),
$$

$\unlhd$ означает $\leqslant$, то оптимальный процесс $\left(x^{*}, u^{*}\right) \in D$ находится из условия

$$
\max _{i \in T \backslash T_{0}} f\left(i, x_{i}^{*}, x_{\pi(i)}^{*}, u_{i}^{*}\right)=\min _{(x, u) \in D} \max _{i \in T \backslash T_{0}} f\left(i, x_{i}, x_{\pi(i)}, u_{i}\right) .
$$

ЗАМЕчАНИЕ 2. Если

$$
\begin{gathered}
P=\{p \in W \mid 0 \leqslant p\}(W \in\{\mathbb{R}, \mathbb{Q}, \mathbb{Z}, \mathbb{N}\}), \\
p \perp q=p+q(\text { либо } p \perp q=p \cdot q)(\forall p, q \in P),
\end{gathered}
$$

$\unlhd$ означает $\leqslant$, то оптимальный процесс $\left(x^{*}, u^{*}\right) \in D$ находится из условия

$$
\begin{gathered}
\sum_{i \in T \backslash T_{0}} f\left(i, x_{i}^{*}, x_{\pi(i)}^{*}, u_{i}^{*}\right)=\min _{(x, u) \in D} \sum_{i \in T \backslash T_{0}} f\left(i, x_{i}, x_{\pi(i)}, u_{i}\right) \\
\left(\text { либо } \prod_{i \in T \backslash T_{0}} f\left(i, x_{i}^{*}, x_{\pi(i)}^{*}, u_{i}^{*}\right)=\min _{(x, u) \in D} \prod_{i \in T \backslash T_{0}} f\left(i, x_{i}, x_{\pi(i)}, u_{i}\right)\right) .
\end{gathered}
$$

ЗАмечАниЕ 3 . В задаче $\mathfrak{A}$ линейно упорядоченная абелева полугруппа $\langle P, \perp, \unlhd\rangle$ может получаться как лексикографическое произведение линейно упорядоченных абелевых полугрупп $\left\langle P_{k}, \perp_{k}, \unlhd_{k}\right\rangle\left(\forall k \in N_{m}\right)(m \in \mathbb{N} \backslash\{1\})$ или, иными словами, определяться из следующих условий:

- $P$ - множество функций

$$
q: N_{m} \longrightarrow \bigcup_{k \in N_{m}} P_{k}
$$

со значениями $q_{k} \in P_{k}\left(\forall k \in N_{m}\right)$, 
$-(p \unlhd q) \Leftrightarrow(p=q) \vee\left(\emptyset \neq K=\left\{k \in N_{m} \mid p_{k} \neq q_{k}\right\}\right) \wedge(e=\min K) \wedge\left(p_{e} \unlhd_{e} q_{e}\right)$ $(\forall p, q \in P)$,

- $(p \perp q)_{k}=p_{k} \perp_{k} q_{k}\left(\forall k \in N_{m}\right)(\forall p, q \in P)$.

ЗАмечАниЕ 4. Если полугруппа $\langle P, \perp, \unlhd\rangle$ определяется по замечанию 3 , $P_{k}=\mathbb{R}\left(\forall k \in N_{m}\right), \unlhd_{k}$ означает $\leqslant\left(\forall k \in N_{m}\right), \perp_{k}$ означает $+\left(\forall k \in N_{m}\right)$ и, кроме того,

$$
S(i, \alpha, \beta)=\{s(i, \alpha, \beta)\}
$$

и

$$
(f(i, s(i, \alpha, \beta), \alpha, \beta))_{k}=f^{k}(i, \alpha, \beta)\left(\forall i \in T \backslash T_{0}\right)(\forall \alpha \in A)(\forall \beta \in B)\left(\forall k \in N_{m}\right),
$$

то оптимальный процесс $\left(x^{*}, u^{*}\right) \in D$ является $m$-оптимальным процессом [2].

2. В общем случае наряду с обозначениями [2]

$$
T_{k}=\{i \in T \mid h(i)=k\}\left(\forall k \in N_{h(T)}\right),
$$

где

$$
h(T)=\max _{i \in T} h(i), \quad h(i)=|\{j \in T \mid j \prec i\}|,
$$

будет использоваться следующее развитие терминов [2]:

- пара $(Y, V)$ называется подходящей, если её составляют функции $Y, V$ со значениями

$$
Y(i) \subset A(\forall i \in T), V(i, \alpha) \subset B(\forall \alpha \in Y(\pi(i)))\left(\forall i \in T \backslash T_{0}\right),
$$

когда $Y(i) \neq \emptyset(\forall i \in T)$;

- для подходящей пары $(Y, V)$ множество $F(Y, V)$ образуют пары $(x, u)$ функций $x, u$ со значениями

$$
x_{i} \in A(\forall i \in T), \quad u_{i} \in B(\forall i \in T)
$$

при ограничениях

$$
\begin{gathered}
x_{i}=u_{i}\left(\forall i \in T_{0}\right), \quad x_{i} \in S\left(i, x_{\pi(i)}, u_{i}\right)\left(\forall i \in T \backslash T_{0}\right), \\
x_{i} \in Y(i)(\forall i \in T), \quad u_{i} \in V\left(i, x_{\pi(i)}\right)\left(\forall i \in T \backslash T_{0}\right) ;
\end{gathered}
$$

- подходящая пара $(Y, V)$ называется $S$-согласованной, если выполнены условия

$$
\emptyset \neq V(i, \alpha)=\{\beta \in V(i, \alpha) \mid \emptyset \neq S(i, \alpha, \beta) \cap Y(i)\}(\forall \alpha \in Y(\pi(i)))\left(\forall i \in T \backslash T_{0}\right) .
$$

3. Следующее утверждение (теорема 1), развивающее теорему 1 [2], даёт характеризацию (необходимые и достаточные условия) разрешимости задачи $\mathfrak{A}$.

Теорема 1. Оптимальный процесс задачи $\mathfrak{A}$ существует, если и толъко если из равенств

$$
X^{\partial}(i)=X(i)(\forall i \in M),
$$




$$
\begin{gathered}
X^{\partial}(i)=\left\{\alpha \in X(i) \mid \emptyset \neq\left\{\beta \in U(j, \alpha) \mid \emptyset \neq S(j, \alpha, \beta) \cap X^{\partial}(j)\right\}(\forall j \in \tau(i))\right\} \\
\left(\forall i \in T_{k-1} \backslash M\right)\left(\forall k \in N_{h(T)}\right)
\end{gathered}
$$

(по уменъшению $k$ ) получаются непустые множества достижимости

$$
X^{\partial}(i)(\forall i \in T) \text {. }
$$

При этих условиях определяемая значениями

$$
X^{\partial}(i) \subset A(\forall i \in T)
$$

функиия $X^{\partial}$ и определяемая значениями

$U^{\partial}(i, \alpha)=\left\{\beta \in U(i, \alpha) \mid \emptyset \neq S(i, \alpha, \beta) \cap X^{\partial}(i)\right\} \subset B\left(\forall \alpha \in X^{\partial}(\pi(i))\right)\left(\forall i \in T \backslash T_{0}\right)$

функиия $U^{\partial}$ составляют $S$-согласованную пару $\left(X^{\partial}, U^{\partial}\right)$, удовлетворяющую равенству $D=F\left(X^{\partial}, U^{\partial}\right)$.

4. Далее предполагается, что оптимальный процесс задачи $\mathfrak{A}$ существует, пара $\left(X^{\partial}, U^{\partial}\right)$ составлена, как указано в теореме 1 , и, следовательно,

$$
\begin{gathered}
D=F\left(X^{\partial}, U^{\partial}\right), \quad \emptyset \neq X^{\partial}(i)(\forall i \in T), \\
S(i, \alpha, \beta) \neq \emptyset \neq U^{\partial}(i, \alpha)=\left\{\beta \in U^{\partial}(i, \alpha) \mid \emptyset \neq S(i, \alpha, \beta) \cap X^{\partial}(i)\right\} \\
\left(\forall \beta \in U^{\partial}(i, \alpha)\right)\left(\forall \alpha \in X^{\partial}(\pi(i))\right)\left(\forall i \in T \backslash T_{0}\right) .
\end{gathered}
$$

Также предполагается, что так называемые значения Беллмана $\mathbf{B}(i, \alpha)$ определены из условий

$$
(i \in M) \Rightarrow \mathbf{B}(i, \alpha)=\min _{\beta \in U^{\partial}(i, \alpha)}^{(\unlhd)} f(i, \psi(S(i, \alpha, \beta)), \alpha, \beta)
$$

$(i \notin M) \Rightarrow \mathbf{B}(i, \alpha)=\min _{\beta \in U^{\partial}(i, \alpha)}^{(\unlhd)}\left(f(i, \psi(S(i, \alpha, \beta)), \alpha, \beta) \perp{\underset{j \in \tau(i)}{\perp}}_{\in} \mathbf{B}(j, \psi(S(i, \alpha, \beta)))\right)$

$\left(\forall \alpha \in X^{\partial}(\pi(i))\left(\forall i \in T_{k}\right)\left(\forall k \in N_{h(T)}\right) \quad\right.$ (индукцией по уменьшению $\left.k\right)$,

где $\psi$ - функция выбора при условиях

$$
\emptyset \neq C \Rightarrow \psi(C) \in C(\forall C \subset A \cup B \cup T)
$$

Следующее утверждение (теорема 2) в определённых пределах характеризует оптимальность процесса задачи $\mathfrak{A}$.

Теорема 2. Процесс $\left(x^{*}, u^{*}\right) \in D$ задачи $\mathfrak{A}$ оптимален, если (и при стабильности $\triangleleft$ относительно $\perp$, только если) одновременно выполнены условия

$$
\mathbf{B}\left(i, x_{\pi(i)}^{*}\right)=f\left(i, x_{i}^{*}, x_{\pi(i)}^{*}, u_{i}^{*}\right) \quad(\forall i \in M)
$$




$$
\begin{aligned}
& \mathbf{B}\left(i, x_{\pi(i)}^{*}\right)=f\left(i, x_{i}^{*}, x_{\pi(i)}^{*}, u_{i}^{*}\right) \perp \underset{j \in \tau(i)}{\perp} \mathbf{B}\left(j, x_{i}^{*}\right) \quad\left(\forall i \in\left(T \backslash T_{0}\right) \backslash M\right), \\
& \perp_{j \in \tau(i)} \mathbf{B}\left(j, x_{i}^{*}\right)=\min _{\alpha \in X^{\partial}(i)}^{(\unlhd)} \underset{j \in \tau(i)}{\perp} \mathbf{B}(j, \alpha)\left(\forall i \in T_{0}\right) .
\end{aligned}
$$

ЗАмечАниЕ 5. В случае замечания 1 (когда нет стабильности $\triangleleft$ относительно $\perp)$ легко строится задача $\mathfrak{A}$ с оптимальным процессом, не удовлетворяющим всем условиям теоремы 2.

Из теоремы 2 получается

СледствиЕ. Значения функиий, составляющих оптимальный процесс $\left(x^{*}, u^{*}\right) \in D$ задачи $\mathfrak{A}$, находит следующий алгоритм динамического программирования (алгоритм $\mathfrak{B})$.

Алгоритм $\mathfrak{B}$. Этот алгоритм образуют описываемые при помощи псевдоязыка Pascal [4] следующие последовательно выполняемые действия (Цикл 1, Цикл 2, Цикл 3):

Цикл 1, находящий элементы $\mathbf{x}(i, \alpha)(\in S(i, \alpha, \mathbf{u}(i, \alpha))), \mathbf{u}(i, \alpha)\left(\in U^{\partial}(i, \alpha)\right)$ из условий

$$
\begin{gathered}
i \in M \Rightarrow \mathbf{B}(i, \alpha)=f(i, \mathbf{x}(i, \alpha), \alpha, \mathbf{u}(i, \alpha)) \\
i \notin M \Rightarrow \mathbf{B}(i, \alpha)=f(i, \mathbf{x}(i, \alpha), \alpha, \mathbf{u}(i, \alpha)) \perp \underset{j \in \tau(i)}{\perp} \mathbf{B}(j, \mathbf{x}(i, \alpha)) \\
\left(\forall \alpha \in X^{\partial}(\pi(i))\left(\forall i \in T \backslash T_{0}\right):\right.
\end{gathered}
$$

for $k:=h(T)$ downto 1 do

for для каждого $i \in T_{k}$ do

for для каждого $\alpha \in X^{\partial}(\pi(i))$ do

begin $\beta^{*}:=\psi\left(U^{\partial}(i, \alpha)\right) ; r^{*}:=f\left(i, \psi\left(S\left(i, \alpha, \beta^{*}\right)\right), \alpha, \beta^{*}\right)$;

if $\tau(i) \neq \emptyset$ then

for для каждого $j \in \tau(i)$ do $r^{*}:=r^{*} \perp \mathbf{B}\left(j, \psi\left(S\left(i, \alpha, \beta^{*}\right)\right)\right)$;

if $U^{\partial}(i, \alpha) \backslash\left\{\beta^{*}\right\} \neq \emptyset$ then

for для каждого $\beta \in U^{\partial}(i, \alpha) \backslash\left\{\beta^{*}\right\}$ do

begin $r:=f(i, \psi(S(i, \alpha, \beta)), \alpha, \beta)$;

if $\tau(i) \neq \emptyset$ then

for для каждого $j \in \tau(i)$ do $r:=r \perp \mathbf{B}(j, \psi(S(i, \alpha, \beta)))$;

end; $b:=\operatorname{not}\left(r^{*} \unlhd r\right)$; if $b$ then begin $r^{*}:=r ; \beta^{*}:=\beta$ end;

end.

$$
\mathbf{B}(i, \alpha):=r^{*} ; \mathbf{x}(i, \alpha):=\psi\left(S\left(i, \alpha, \beta^{*}\right)\right) ; \mathbf{u}(i, \alpha):=\beta^{*}
$$

Цикл $2,\left(\forall i \in T_{0}\right)$ получающий $x_{i}^{*}, u_{i}^{*}$, используя

$$
\mathbf{B}(j, \alpha)\left(\forall \alpha \in X^{\partial}(\pi(j))\right)\left(\forall j \in T_{1}\right):
$$

for для каждого $i \in T_{0}$ do 
begin $\Delta:=\tau(i) \backslash\{\psi(\tau(i))\} ; \alpha^{*}:=\psi\left(X^{\partial}(i)\right) ; r^{*}:=\mathbf{B}\left(\psi(\tau(i)), \alpha^{*}\right)$; if $\Delta \neq \emptyset$ then

for для каждого $j \in \Delta$ do $r^{*}:=r^{*} \perp \mathbf{B}\left(j, \alpha^{*}\right)$;

if $X^{\partial}(i) \backslash\left\{\alpha^{*}\right\} \neq \emptyset$ then

for для каждого $\alpha \in X^{\partial}(i) \backslash\left\{\alpha^{*}\right\}$ do

begin $r:=\mathbf{B}(\psi(\tau(i)), \alpha)$;

if $\Delta \neq \emptyset$ then

for для каждого $j \in \Delta$ do $r:=r \perp \mathbf{B}(j, \alpha)$;

end;

$b:=\operatorname{not}\left(r^{*} \unlhd r\right) ;$ if $b$ then begin $r^{*}:=r ; \alpha^{*}:=\alpha$ end

end.

$u_{i}^{*}:=x_{i}^{*}:=\alpha^{*}$

Цикл 3, $\left(\forall i \in T \backslash T_{0}\right)$ получающий $x_{i}^{*}, u_{i}^{*}$ с использованием $x_{i}^{*}\left(\forall i \in T_{0}\right)$, найденных циклом 2 , и использованием $\left(\forall \alpha \in X^{\partial}(\pi(i))\left(\forall i \in T \backslash T_{0}\right)\right.$ элементов $\mathbf{x}(i, \alpha), \mathbf{u}(i, \alpha)$, найденных циклом 1 :

for $k:=1$ to $h(T)$ do for для каждого $i \in T_{k}$ do

begin

$x_{i}^{*}:=\mathbf{x}\left(i, x_{\pi(i)}^{*}\right) ; u_{i}^{*}:=\mathbf{u}\left(i, x_{\pi(i)}^{*}\right)$ end.

ЗАмечание 6 . В случае замечания 1 алгоритм $\mathfrak{B}$ модифицируется по правилам

$$
\begin{gathered}
(b:=\operatorname{not}(p \unlhd q)) \Leftrightarrow(b:=\operatorname{not}(p \leqslant q))(\forall p, q \in P), \\
(p:=p \perp q) \Leftrightarrow(p:=\max (p, q))(\forall p, q \in P) .
\end{gathered}
$$

ЗАмечание 7 . В случае замечания 2 , алгоритм $\mathfrak{B}$ модифицируется по правилам

$$
\begin{gathered}
(b:=\operatorname{not}(p \unlhd q)) \Leftrightarrow(b:=\operatorname{not}(p \leqslant q))(\forall p, q \in P), \\
(p:=p \perp q) \Leftrightarrow(p:=p+q(p:=p \cdot q))(\forall p, q \in P) .
\end{gathered}
$$

ЗАмечание 8 . В случае замечания 3 алгоритм $\mathfrak{B}$ модифицируется по правилам

$$
\begin{gathered}
(b:=\operatorname{not}(p \unlhd q)) \Leftrightarrow \\
\left(b:=\text { false } k:=1 ; \text { while }\left(p_{k}=q_{k}\right) \wedge(k \leqslant m) \operatorname{do} k:=k+1 ;\right. \\
\left.\quad \text { if } k \leqslant m \text { then } b:=\operatorname{not}\left(p_{k} \unlhd_{k} q_{k}\right)\right)(\forall p, q \in P), \\
(p:=q) \Leftrightarrow\left(\text { for } k:=1 \text { to } m \text { do } p_{k}:=q_{k}\right)(\forall p, q \in P), \\
(p:=p \perp q) \Leftrightarrow\left(\text { for } k:=1 \text { to } m \text { do } p_{k}:=p_{k} \perp_{k} q_{k}\right)(\forall p, q \in P) .
\end{gathered}
$$

ЗАмечАние 9. В случае замечания 4 модифицированный по правилам замечания 8 алгоритм $\mathfrak{B}$ получает $m$-оптимальный процесс проще алгоритма [2] за счет неиспользования, нехранения и неполучения $s$-согласованных пар $\left(X^{k}, U^{k}\right)\left(\forall k \in N_{m}\right)$, даваемых теоремой 2 [2].

\section{ORCID}

Валерий Гаврилович Овчинников: http://orcid.org/0000-0003-2234-5010 


\title{
БИБЛИОГРАФИЧЕСКИЙ СПИСОК
}

1. Овчинников В. Г. Алгоритмы динамического программирования оптимальных и близких к ним процессов / Труды пятой Всероссийской научной конференции с международным участием (29-31 мая 2008 г.). Часть 4: Информационные технологии в математическом моделировании / Матем. моделирование и краев. задачи. Самара: СамГТУ, 2008. C. $107-112$.

2. Овчинников В. Г. К алгоритмам динамического программирования оптимальных процессов // Вестн. Сам. гос. техн. ун-та. Сер. Физ.-мат. науки, 2012. №3(28). С. 215-218. doi: $10.14498 /$ vsgtu1102.

3. Фор Р., Кофман А., Дени-Папен М. Современная математика. М.: Мир, 1966. 271 с.

4. Ахо А. В., Хопкрофт Д. Э., Ульман Д. Д. Структурь даннъх и алгоритмы. М.: Вильямс, 2009. 400 с.

Поступила в редакцию $04 / \mathrm{II} / 2016$;

в окончательном варианте - 22/II/2016;

принята в печать - 26/II/2016.

Vestn. Samar. Gos. Techn. Un-ta. Ser. Fiz.-mat. nauki

[J. Samara State Tech. Univ., Ser. Phys. \& Math. Sci.], 2016, vol. 20, no. 1, pp. $158-166$

ISSN: 2310-7081 (online), 1991-8615 (print)

doi: http://dx.doi.org/10.14498/vsgtu1473

MSC: 90C39

\section{ON DYNAMIC PROGRAMMING ON THE VALUES IN THE SEMIGROUP}

\section{G. Ovchinnikov}

Samara State Technical University,

244, Molodogvardeyskaya st., Samara, 443100, Russian Federation.

\begin{abstract}
For not considered previously discrete optimal control problem with target function values in a linearly ordered Abelian semigroup given characterization of the solvability and on its basis the algorithm seeks optimal process with the help of delivering Bellman values elements of limiting sets. We mark the modifications to this algorithm, when

1) $P$ is nonempty subset of numbers with the natural ordering and the operation producing the maximum of two numbers;

2) $P$ is set of nonnegative numbers with the natural ordering and the addition (or multiplication);

3) $P$ is lexicographical product of $m$ (not less than two) linearly ordered Abelian semigroups;
\end{abstract}

(C) 2016 Samara State Technical University.

Please cite this article in press as:

Ovch innikov V. G. On dynamic programming on the values in the semigroup, Vestn. Samar. Gos. Tekhn. Univ., Ser. Fiz.-Mat. Nauki [J. Samara State Tech. Univ., Ser. Phys. \& Math. Sci.], 2016, vol. 20, no. 1, pp. 158-166. doi: 10.14498/vsgtu1473. (In Russian)

Author Details:

Valery G. Ovchinnikov (ovg.samara@mail.ru), Senior Lecturer, Dept. of Oil and Gas Fields Development. 
4) $P$ is lexicographic product of $m$ (not less than two) sets of real numbers with the natural ordering and the addition, and this algorithm gets $m$ optimal process easier than the previous author's algorithm.

Keywords: linearly ordered Abelian semigroup, discrete optimal control, optimal process, delivering Bellman values elements of limiting sets, dynamic programming, lexicographical products, algorithms.

\section{ORCID}

Valery G. Ovchinnikov: http://orcid.org/0000-0003-2234-5010

\section{REFERENCES}

1. Ovchinnikov V. G. Algorithms of dynamic programming for optimal and similar processes, Proceedings of the Fifth All-Russian Scientific Conference with international participation (29-31 May 2008). Part 4, Matem. Mod. Kraev. Zadachi. Samara, Samara State Technical Univ., 2008, pp. 107-112 (In Russian).

2. Ovchinnikov V. G. On the algorithms of dynamic programming for optimal processes, Vestn. Samar. Gos. Tekhn. Univ. Ser. Fiz.-Mat. Nauki, 2012, vol.3(28), pp. 215-218 (In Russian). doi: $10.14498 /$ vsgtu1102.

3. Faure R., Kaufmann A., Denis-Papin M. Manuale di Matematica. Milano, ISEDI, 1975 (In Italian).

4. Aho A. V., Hopcroft J. E., Ullman J. D., Data Structures and Algorithms, AddisonWesley Series in Computer Science and Information Processing. Reading, Massachusetts, etc., Addison-Wesley, 1983, xi+427 pp.

Received 04/II/2016;

received in revised form $22 / \mathrm{II} / 2016$;

accepted 26/II/2016. 